
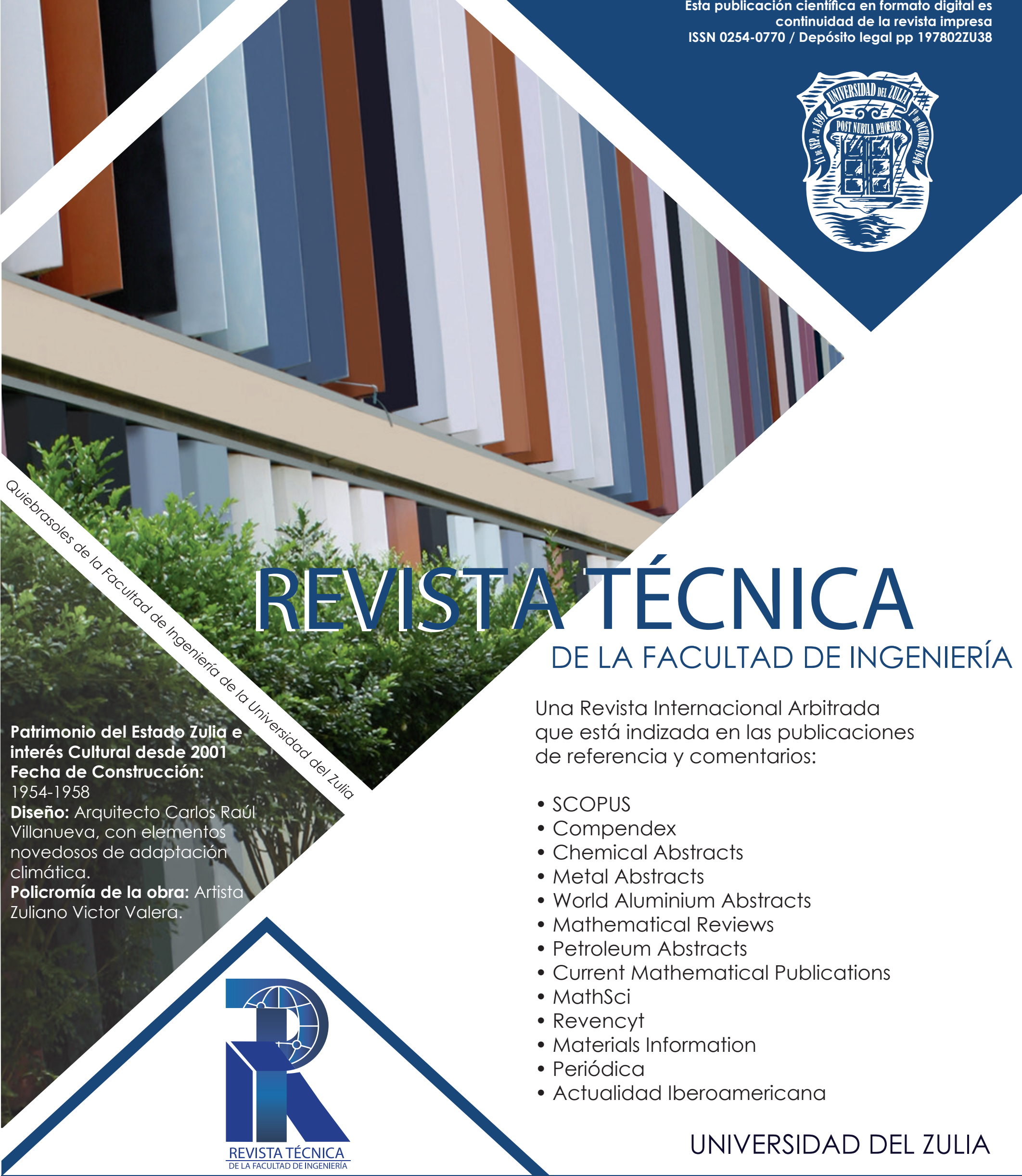
Rev. Téc. Ing. Univ. Zulia. Vol. 42, No. 3, 2019, 118-125

\title{
Drag coefficients of lattice towers with VHF antennas on wind tunnel
}

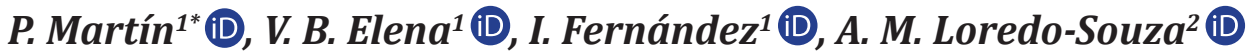 \\ ${ }^{1}$ Centro de Estudios de la Construcción y Arquitectura Tropical (CECAT), Facultad de Ingeniería Civil, Universidad \\ Tecnológica de La Habana José Antonio Echeverría (CUJAE). Marianao, Apartado 19390, La Habana, Código Postal 11500 , \\ Cuba \\ ${ }^{2}$ Laboratorio de Aerodinámica de las Construcciones (LAC), Universidade Federal do Rio Grande do Sul (UFRGS), Rua \\ Ferreira Viana, 14/5, Porto Alegre, RS, CEP: 90.670-100, Brazil. \\ *Autor de Contacto: patriciamr@civil.cujae.edu.cu \\ https://doi.org/10.22209/rt.v42n3a03 \\ Recepción: 28/02/2019 | Aceptación: 23/05/2019 | Publicación: 01/09/2019
}

\begin{abstract}
This article presents some of the principal aspects to be taken into account for undertaking wind tunnel static tests on lattice towers. This work describes a test performed on a tower segment with square cross section considering VHF antennas, as a study case. This paper is aimed at obtaining drag coefficients for the tower and the antennas independently and the increase in the drag coefficient and interference factors considering the tower and antennas at the same time. The results presented show values for interference factors higher than 1 for $45^{\circ}$ wind direction, indicating the necessity of calculating the wind force for the tower and the antennas at the same time and not independently overlapping the drag coefficient values since this may result in a sub-estimation of the wind action on the structure.
\end{abstract}

Keywords: Aerodynamics; Antennas; Lattice Towers; Static Tests; Wind Tunnel

\section{Coeficientes de arrastre de torres reticuladas con antenas VHF mediante ensayo en túnel de viento}

\section{Resumen}

En el presente artículo se exponen algunos de los principales aspectos a que se deben considerar para el desarrollo de ensayos estáticos a torres reticuladas con el empleo de túneles de viento. Se describe, como caso de estudio, un ensayo realizado a un módulo de torre de sección cuadrada con presencia de antenas VHF, con el objetivo de obtener los coeficientes de arrastre para el módulo y para las antenas de forma independiente, así como los factores de interferencia para el módulo de torre con las antenas VHF incorporadas ensayados de forma simultánea. Los resultados muestran valores de factores de interferencia mayores de uno para la dirección de viento a 45 grados lo que permite concluir que es necesario calcular la carga de viento de la torre reticulada con las antenas colocadas y no de forma independiente, superponiendo los valores de coeficientes de arrastre, pues se subestima la acción del viento sobre la estructura.

Palabras clave: Aerodinámica; Antenas; Ensayos Estáticos; Torres Reticuladas; Túnel de Viento. 


\section{Introducción}

El análisis del efecto del viento sobre las torres de telecomunicaciones adquiere gran importancia debido al fallo más de más 50 torres de telecomunicaciones en los últimos 20 años en Cuba durante el paso de los huracanes. Uno de los aspectos trascendentales en el análisis de estas estructuras es la correcta determinación de la carga de viento sobre la torre y sus antenas. Los principales tipos de antenas que soportan las torres de telecomunicaciones son UHF, VHF y parabólicas.

La carga de viento depende de muchos factores que pueden agruparse en parámetros meteorológicos y parámetros aerodinámicos o de forma. Los parámetros meteorológicos actúan modificando la velocidad de viento, mientras que los parámetros aerodinámicos modifican el valor de la presión ejercida por el viento sobre la estructura, ya que tienen en cuenta la resistencia aerodinámica de los objetos expuestos al viento. La presencia de las antenas modifica el flujo del aire que pasa sobre las torres, por tanto, modifica los parámetros aerodinámicos de estas estructuras. La principal herramienta en la obtención de los parámetros aerodinámicos, en particular los coeficientes de fuerza aerodinámicos, ha sido a lo largo de la historia, los túneles de viento. En el cálculo de la carga de viento sobre las torres de telecomunicaciones y sus antenas, el coeficiente de fuerza aerodinámico que se utiliza es el coeficiente de arrastre que representa el valor adimensional de la fuerza de arrastre. Esta fuerza es definida como la fuerza que un fluido en movimiento ejerce sobre un cuerpo en la dirección del flujo [1] y es debida a los efectos combinados de la presión y de la fuerza de rozamiento en la dirección del flujo, por tanto, los coeficientes de fuerza aerodinámicos asociados a la fuerza de arrastre (coeficientes de arrastre) tienen en cuenta tanto los efectos de presión como los de rozamiento.

La carga de viento que ejercen las antenas sobre las torres puede determinarse por dos vías fundamentales. La primera vía consiste en el cálculo de la carga de viento de la antena como una fuerza adicional sobre la torre a partir de utilizar un coeficiente de arrastre de la antena sin considerar que se encuentra colocada sobre la torre; esta forma es la más frecuente ya que los valores de las fuerzas de arrastre de las antenas se pueden encontrar en los catálogos de los fabricantes que son obtenidos a partir de realizar ensayos en túneles de viento a las antenas de forma independiente. En la segunda vía se tiene en cuenta el efecto de la antena cuando se encuentra colocada sobre la torre a partir de modificar el valor del coeficiente de arrastre de la antena aislada por un factor de interferencia, que relaciona el coeficiente de arrastre de la antena independiente y del módulo de torre con la antena incorporada; obtenido mediante ensayos en túnel de viento. Varios trabajos publicados sobre la influencia de la presencia de las antenas en los valores de coeficientes de arrastre de las torres de telecomunicaciones y las propuestas de formulaciones para el cálculo de factores de interferencias han estado dirigidos fundamentalmente a las tipologías de antenas parabólicas [2-5]; sin embargo los valores de los coeficientes de arrastre producto de la presencia de las antenas VHF y su influencia cuando están colocadas sobre las torres de celosía no han sido encontrados en la literatura. En este trabajo se analiza un ensayo realizado a un módulo de torre de sección cuadrada con presencia de antenas VHF, con el objetivo de obtener sus coeficientes de arrastre y los factores de interferencia producto de las antenas.

\section{Metodología}

Los ensayos estáticos y dinámicos son principalmente los dos tipos de pruebas que se realizan a las estructuras en túneles de viento para la determinación de su comportamiento aerodinámico. A partir de los ensayos estáticos se obtienen los coeficientes de arrastre y sustentación. El segundo tipo de ensayos se emplea fundamentalmente para profundizar en el comportamiento dinámico de estructuras frente al viento con fines diversos, para lo cual deben ser confeccionados modelos aero-elásticos donde se reproduzcan las características dinámicas: masa, rigidez y amortiguamiento. El objetivo de este trabajo es obtener coeficientes de arrastre, por lo que se decide hacer uso de los ensayos estáticos y para esto se emplean generalmente modelos a escala reducida en lugar del prototipo a escala natural debido a su elevado costo. En estos casos para poder afirmar que los resultados del modelo a escala son equivalentes a los resultados del prototipo es necesario cumplir con los requerimientos básicos de semejanza. En estos ensayos se reproducen las características geométricas y la forma del flujo alrededor del modelo para obtener los coeficientes de arrastre y sustentación, así como los coeficientes de presión.

El primer paso en el proceso de realización de ensayos estáticos a torres reticuladas en túnel de viento es la determinación de las características de los modelos. Estas características se obtienen de la aplicación del método de análisis dimensional a partir de aplicar el Teorema de Pi-Buckingham para determinar los grupos adimensionales y luego se aplican las leyes de semejanza correspondientes. La principal dificultad que suele encontrarse en la aplicación de las leyes de semejanza es que no se puede igualar el número de Reynolds del modelo y del prototipo. En estos casos, la semejanza que se obtendrá entre el modelo y el prototipo se llama semejanza incompleta y a los modelos se les conoce como modelos distorsionados [1]. La solución a este problema radica en la relación que existe entre el coeficiente de arrastre y el número de Reynolds, ya que en determinadas estructuras no hay dependencia entre estos números adimensionales a partir de determinado valor del número de Reynolds [1], por tanto si se garantiza que los modelos presenten números de Reynolds mayores que el número de Reynolds límite, se garantiza que exista semejanza entre las fuerzas aerodinámicas que actúan entre estos y los prototipos. Para el diseño de los modelos estáticos de 
las torres y las antenas VHF se investigó cuál era el valor límite del número de Reynolds en cada caso.

El número de Reynolds en los elementos de perfiles angulares en el modelo debe ser mayor que 2200 según los planteamientos de Vickery [6] y Holdo [7]. Para cumplir este criterio se calcula la longitud mínima del ancho de los perfiles () correspondiente al valor límite del número de Reynolds (0 teniendo en cuenta la velocidad de viento en el túnel (), la viscosidad dinámica ( ) y la densidad del aire (). El espesor de los perfiles generalmente no necesita ser escalado ya que no es una dimensión que genere un áera expuesta signficativa frente al viento, además en los ensayos estáticos no es necesario reproducir la masa, ni la rigidez del protototipo.

En el caso de torres construidas con perfiles tubulares la forma del flujo depende de las condiciones de velocidad del flujo, turbulencia, viscosidad, esto significa que depende del número de Reynolds () y del acabado superficial del tubo, ya que la dependencia entre el coeficiente de arrastre y el número de Reynolds puede variar si la superficie es rugosa o lisa. Para el análisis de la dependencia del número de Reynolds deben ser calculados los números de Reynolds del prototipo y del modelo. Para el cálculo del número de Reynolds del prototipo se recomienda emplear la velocidad de proyecto (velocidad normada en el código de viento correspondiente según la ubicación de la torre en estudio). Según Schlichting referenciado por Fox and McDonald's [8], los valores de los números de Reynolds obtenidos del modelo y del prototipo deben encontrarse en el rango entre $10^{3}$ y $2 \times 10^{5}$ que corresponden a la zona donde los coeficientes de arrastre son constantes en las secciones tubulares, siendo recomendable trabajar en estos intervalos.

Otros aspectos que deben considerarse en la elaboración de los modelos son las dimensiones del túnel, las características de los materiales para la construcción y la razón de bloqueo, definida como la razón, en porcentaje, entre el área del objeto a ensayar y el área de la sección transversal de ensayo en el túnel. Las dimensiones del túnel se deben tener en cuenta con el objetivo de garantizar el aprovechamiento máximo de su espacio físico asociado con la mayor longitud posible de los modelos a fin de lograr un flujo de aire apropiado. Los materiales para la construcción de modelos estáticos deben ser rígidos con el objetivo de no permitir deformaciones y que los valores que se obtengan estén asociados únicamente a la fuerza de arrastre del viento sobre el modelo. En un túnel de viento sus paredes generan interferencia del flujo alrededor del modelo, esta interferencia depende de la razón de bloqueo, definida como el cociente del área máxima de la sección transversal del modelo (en las celosías corresponde al área proyectada del modelo) y el área de la sección transversal del túnel de viento [9]. Si la razón de bloqueo es menor del 5\% [9] no es necesario hacer correcciones en los resultados de los coeficientes de arrastre obtenidos de los ensayos.

Posterior a la caracterización y construcción de los modelos estáticos está la definición de las direcciones de viento a ensayar. Según las indicaciones de las normas de torres de telecomunicaciones $[10,11]$ las direcciones que deben analizarse para estructuras de sección cuadrada son $0^{\circ}$ y $45^{\circ}$ y para torres de sección triangular recomiendan analizar $0^{\circ}$, $60^{\circ}$ y $90^{\circ}$. Cuando se trata de torres que presentan aditamentos que puedan modificar su simetría respecto a los ángulos de incidencia del viento, como es el caso de las antenas en las torres de telecomunicaciones, se debe realizar un estudio particular e incrementar las direcciones de viento a considerar. Se recomienda para las torres de sección cuadradas incrementar cada $45^{\circ}$ y y para torres triangulares cada $30^{\circ}$, máximo $60^{\circ}$.

El siguiente paso en este proceso son las mediciones propiamente. Las magnitudes medidas en un túnel de viento para la determinación de los coeficientes de arrastre son: la velocidad del viento, la fuerza de arrastre, la presión atmosférica y la temperatura.

El cálculo de la velocidad de viento en el túnel antes de incidir en el modelo se realiza a partir de la ecuación de Bernoulli, para lo cual es necesario realizar mediciones de diferencia de presión entre dos secciones específicas del túnel. Generalmente se utiliza un manómetro digital y mediciones de temperatura y presión atmosférica del aire en su interior en el instante de cada uno de los ensayos. Además, se utilizan un termómetro y un barómetro digital para la medición de la temperatura y de la presión atmosférica respectivamente. El valor de la velocidad final se obtiene de calcular la media de todas las muestras adquiridas durante cada adquisición de datos durante los ensayos.

Para determinar la acción del viento sobre las torres de celosía se divide la estructura de la torre en tramos reticulados (módulos) y se determinan las fuerzas de arrastre sobre cada parte. La fuerza de arrastre se obtiene por medio de una balanza de fuerza ubicada por debajo del piso del túnel y acoplada a una base donde son instalados los módulos de la torre.

Cuando se trata con procesamiento de señales analógicas, como son las series de velocidad y de fuerza registradas por el manómetro y la balanza de fuerzas respectivamente, es necesario definir algunos aspectos asociados al proceso de adquisición de datos. Los parámetros que definen la adquisición de datos de una placa A/D son: a) tasa de muestreo $\left(f_{a}\right)$, determina el intervalo de tiempo en el cuál las muestras consecutivas de la señal analógica serán adquiridas; b) número de muestras $\left(N_{a}\right)$, determina el número de puntos de la serie temporal. Estos dos parámetros determinan el tiempo de adquisición $\left(t_{a}\right)$, necesario para digitalizar la 
serie temporal. De forma general, el tiempo de muestreo o adquisición debe fijarse a través de un conocimiento previo del fenómeno a registrar, pues es necesario que el intervalo que se precise sea capaz de incluir las principales características de ese fenómeno.

El proceso de obtención de los coeficientes de arrastre en un módulo de torre de celosía con presencia de antenas VHF a partir de un ensayo en un túnel de viento teniendo en cuenta los elementos mencionados previamente se describe a continuación.

El módulo de torre a ensayar se corresponde con el último tramo de una torre de telecomunicaciones de tipo autosoportada de sección cuadrada, conformada por perfiles angulares metálicos. Se escogió este tramo pues es donde el prototipo a escala real, presenta antenas VHF colocadas en cada cara de la torre (ver Figura 1).

a)

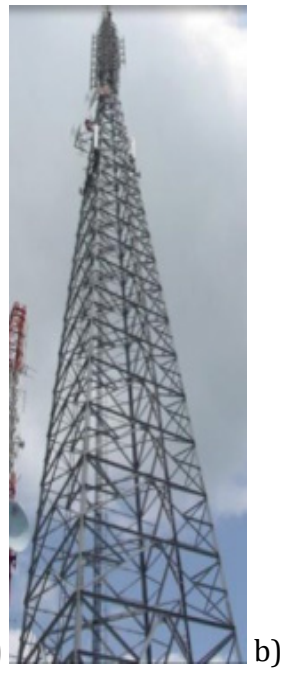

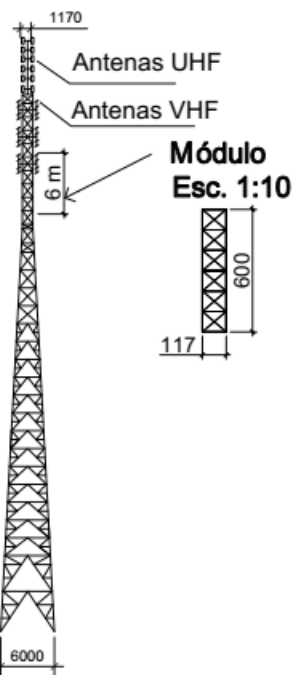

Figura 1. a) Foto del prototipo de torre autosoportada con antenas VHF, b) Esquema de la ubicación de las antenas VHF y del módulo de la torre seleccionado.

Para la determinación de las dimensiones de los modelos estáticos del módulo de la torre y las antenas VHF se investigó cuál era el valor límite del número de Reynolds en cada caso. De acuerdo con lo expuesto anteriormente, se calculó la longitud mínima que debe tener el ancho de los perfiles que garantice un número de Reynolds mayor que $2200[6,7]$, además se tuvieron en cuenta los siguientes datos: velocidad en el túnel $(V) 15 \mathrm{~m} / \mathrm{s}$, viscosidad dinámica del aire $(\mu) 1,8 \times 10^{5} \mathrm{~N} \mathrm{~s} / \mathrm{m}^{2} \mathrm{y}$ densidad del aire ( $\rho$ ): $1,205 \mathrm{~kg} / \mathrm{m}^{3}$. Los valores de viscosidad dinámica y densidad del aire fueron seleccionados para una temperatura de $20^{\circ} \mathrm{C}$. Como resultado de este análisis, la longitud mínima del ancho de los perfiles angulares en el modelo debe ser mayor de $1 \mathrm{~mm}$, lo que significa que se puede aplicar cualquier escala geométrica que garantice esta condición. La escala escogida para la construcción del modelo fue 1:10.

La Tabla 1 muestra las características de los perfiles del prototipo de la torre y las características de los perfiles del modelo, así como los valores obtenidos del número de Reynolds en el modelo donde se corrobora que en todos los casos es mayor que 2200. El modelo de la torre fue construido con perfiles de madera, la Figura 2a muestra fotos de su proceso de construcción. El área del módulo de la torre ensayado fue de $0,033 \mathrm{~m}^{2}$ y el área de la sección transversal del túnel donde fue realizado el ensayo es de $1.12 \mathrm{~m}^{2}$, por tanto la razón de bloqueo obtenida para el modelo fue del $3 \%$ y no hubo que realizar correcciones en los valores de las fuerzas de arrastre.

Las antenas VHF están conformadas por una celosía de elementos cilíndricos, a la cual se encuentran fijados los dipolos que también son elementos cilíndricos lisos (ver Figura 2b). El diámetro de los cilindros de la celosía es de $12 \mathrm{~mm}$ y el de los dipolos es de $50 \mathrm{~mm}$. Las características geométricas de esta antena fueron obtenidas del catálogo de la empresa RYMSA [12]. La escala utilizada para la construcción del modelo de las antenas VHF fue 1:10 porque coincide con la escala utilizada en el modelo de la torre. La Tabla 2 muestra los números de Reynolds calculados para el modelo y el prototipo de las antenas VHF, fue verificado que los Reynolds calculados para el modelo y el prototipo de las antenas VHF, se encontraran en el rango entre $10^{3}$ y $2 \times 10^{5}$ que es la zona donde permanecen constantes los coeficientes de arrastre para cilindros lisos según Schlichting referenciado por Fox and McDonald's [8], lo cual indica que se puede aplicar la escala geométrica definida anteriormente de 1:10. Las velocidades utilizadas para el cálculo del número de Reynolds del prototipo fueron las correspondientes a la velocidad de proyecto considerando la ubicación de la torre en dos regiones diferentes de Cuba, Occidente y

Tabla 1. Características del modelo y prototipo del módulo de torre.

\begin{tabular}{|c|c|c|c|c|c|c|c|c|}
\hline \multicolumn{4}{|c|}{ Prototipo } & & \multicolumn{4}{|c|}{ Modelo } \\
\hline $\begin{array}{l}\text { Altura del } \\
\text { módulo } \\
(\mathrm{mm})\end{array}$ & $\begin{array}{l}\text { Tipo de } \\
\text { Elemento }\end{array}$ & $\begin{array}{l}\text { Sección } \\
\text { del perfil } \\
\text { angular }\end{array}$ & $\begin{array}{l}\text { Ancho del } \\
\text { perfil L } \\
(\mathrm{mm})\end{array}$ & Escala & $\begin{array}{c}\text { Altura del } \\
\text { módulo (mm) }\end{array}$ & $\begin{array}{l}\text { Ancho del } \\
\text { perfil L } \\
(\mathrm{mm})\end{array}$ & $\begin{array}{l}\text { Velocidad } \\
\quad(\mathrm{m} / \mathrm{s})\end{array}$ & $\begin{array}{c}\text { No } \\
\text { Reynolds }\end{array}$ \\
\hline \multirow[t]{2}{*}{6000} & $\begin{array}{l}\text { Tranques y } \\
\text { diagonales }\end{array}$ & $50 \times 5$ & 50 & \multirow[t]{2}{*}{$1: 10$} & \multirow[t]{2}{*}{600} & 5 & 15 & 5021 \\
\hline & Columnas & $90 \times 9$ & 90 & & & 9 & 15 & 9038 \\
\hline
\end{tabular}


Oriente. Estos valores de velocidad de proyecto fueron obtenidos a partir de los valores de presión de viento calculados en el punto de localización de las antenas VHF sobre la torre y considerando todos los coeficientes correspondientes que establece la norma de viento [13], excepto el coeficiente de forma. Los materiales usados para la construcción de los modelos de las antenas VHF fueron varillas de soldar para los dipolos y los cilindros de la celosía, y angulares de madera para la zona exterior de la celosía, ver Figura 2c. $0^{\circ}$ (perpendicular a una cara) y $45^{\circ}$ (oblicua a una cara). La dirección $0^{\circ}$ es la correspondiente a la ubicación frontal de los modelos frente a la entrada de flujo de aire del túnel de viento.

El túnel de viento empleado para los ensayos fue el Túnel de Viento "Prof. Joaquim Blessmann" del Laboratorio de Aerodinámica de las Construcciones (LAC) de la Universidad Federal de Río Grande del Sur (UFRGS), Brasil [14].

Tabla 2. Características del modelo y prototipo de los elementos componentes de las antenas VHF.

\begin{tabular}{|c|c|c|c|c|c|}
\hline \multicolumn{3}{|c|}{ Prototipo } & \multirow[b]{2}{*}{ Escala } & \multicolumn{2}{|c|}{ Modelo } \\
\hline $\begin{array}{l}\text { Diámetro } \\
\text { cilindros } \\
(\mathrm{mm})\end{array}$ & $\begin{array}{l}\text { Nro. Reynolds } \\
\text { cilindros (para } \\
\mathrm{V}=55 \mathrm{~m} / \mathrm{s} \text { ) }\end{array}$ & $\begin{array}{l}\text { Nro. Reynolds } \\
\text { cilindros (para } \\
\mathrm{V}=65 \mathrm{~m} / \mathrm{s} \text { ) }\end{array}$ & & Diámetro cilindros (mm) & $\begin{array}{c}\text { Nro. Reynolds } \\
\text { cilindros (para } \mathrm{V}=15 \\
\mathrm{~m} / \mathrm{s} \text { ) }\end{array}$ \\
\hline 12 (malla) & 44183 & 52217 & $1: 10$ & 1,2 & 1205 \\
\hline 50 (dipolo) & 184097 & 217569 & $1: 10$ & 5 & 5021 \\
\hline
\end{tabular}

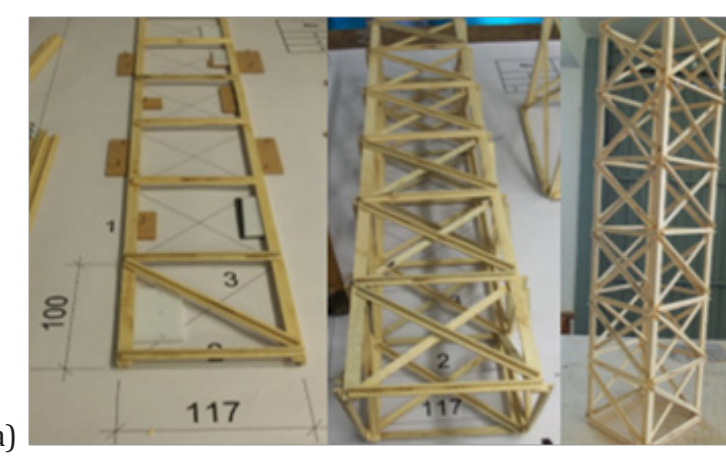

b)

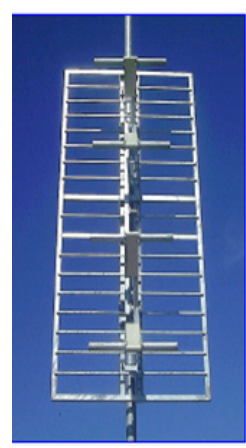

c)

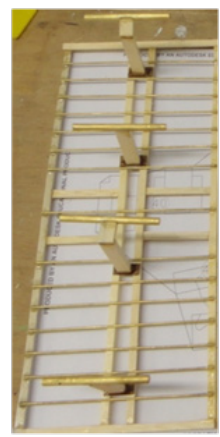

Figura 2. a) Imágenes de la construcción del módulo de torre a ensayar en el túnel de viento b) Antena VHF prototipo c) modelo.

La Figura 3 muestra los tres tipos de ensayos realizados para obtener las fuerzas de arrastre de los modelos de la torre y las antenas: a) módulo de la torre sin antenas, b) antena VHF independiente, c) módulo de la torre con cuatro antenas VHF colocadas en cada una de las caras. Las direcciones de viento ensayadas fueron de
Los ensayos fueron realizados con un perfil simulado de velocidades uniforme, con dos intensidades de turbulencia, clasificadas como intensidad de turbulencia 1 (IT1) e intensidad de turbulencia 2 (IT2). El perfil turbulento de velocidades fue generado a partir de la utilización de una malla. Los valores de intensidad de turbulencia generados fueron de $0,2 \%$ para IT 1 y $12 \%$ para IT2. La velocidad durante los ensayos fue monitoreada por las mediciones de presión entre dos puntos dentro del túnel de viento proporcionadas por el manómetro digital MANOAIR 500. La frecuencia de adquisición de los datos fue de $4 \mathrm{~Hz}$ y el tiempo total de adquisición en cada ensayo fue de $16 \mathrm{~s}$. La presión atmosférica y la temperatura fueron medidas para cada ensayo utilizando el barómetro digital marca NOVUS/HUBA y un termómetro digital respectivamente.

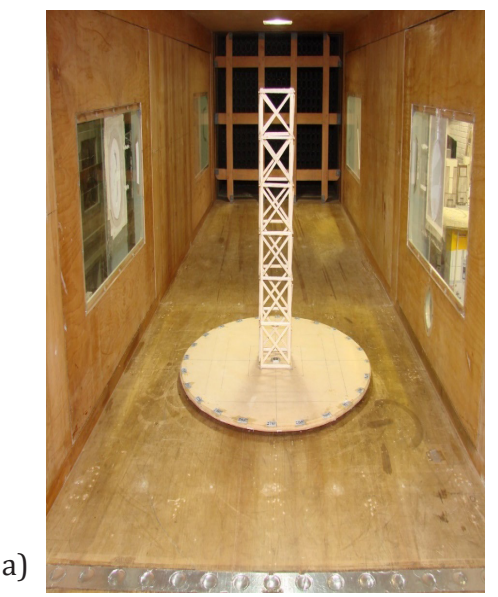



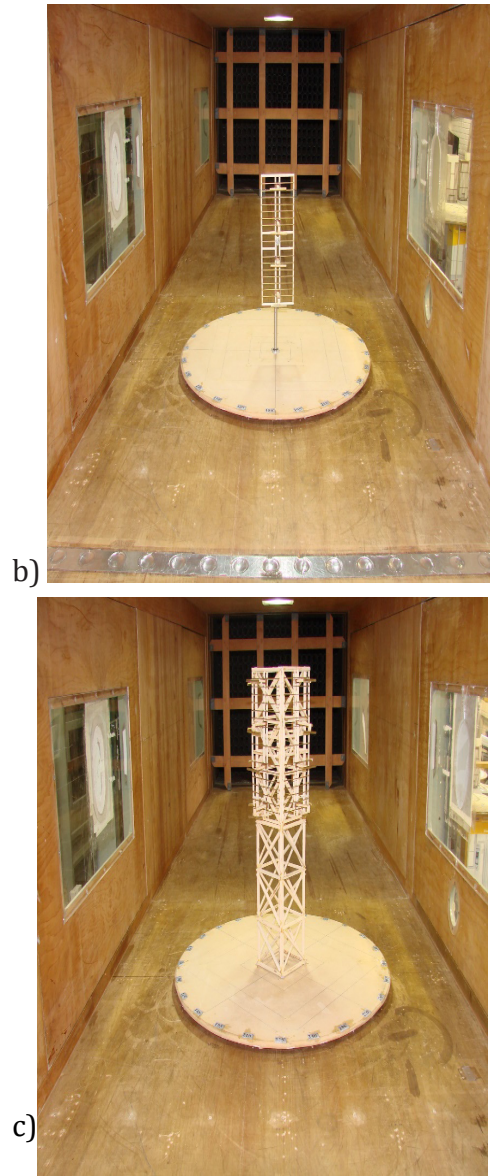

Figura 3. Modelos sobre la mesa de ensayo dentro del túnel de viento, a) módulo de torre sin antenas, b) modelo de antena VHF, c) módulo de torre con antenas VHF colocadas en cada cara.

Para la medición de la fuerza de arrastre los modelos fueron colocados sobre una mesa circular giratoria ubicada sobre el piso del túnel de viento. En el centro de la mesa se fijó una balanza de fuerza unidireccional. Con el objetivo de obtener mayor precisión en las medidas experimentales, se resta a la fuerza de arrastre total medida la fuerza de arrastre generada por la mesa giratoria. Se realizaron dos calibraciones diarias, una en el inicio y otra en el final de la serie de ensayos, con el objetivo de garantizar la normalidad de funcionamiento de las células de medición; aunque se establece un único coeficiente de calibración, que se toma como promedio de las dos calibraciones diarias.

La balanza de fuerza unidireccional utilizada fue construida en el Laboratorio de Aerodinámica de las Construcciones (LAC) de la UFRGS, Brasil. Esta balanza unidireccional está acoplada con extensómetros y se encuentra constituido por dos placas rígidas horizontales, unidas por cuatro láminas verticales de acero inoxidable. La placa inferior está rígidamente acoplada a un trípode fijo sobre el piso de las instalaciones del túnel de viento y la placa superior está acoplada a la mesa giratoria, la cual se desplaza en conjunto con los modelos en el sentido del flujo en proporción con la fuerza de arrastre aerodinámica actuante. Con el objetivo de aumentar la confiabilidad de los resultados de las fuerzas de arrastre obtenidos se utilizó en la construcción de la balanza un sistema redundante constituido por dos pares de láminas cada uno a través de dos puentes completos de Wheatstone instrumentados con cuatro extensómetros cada uno, denominados célula de barlovento y célula de sotavento. La resolución de la balanza utilizada fue de 0,41 g. El sistema de adquisición utilizado para obtener los datos provenientes de la balanza está compuesto por una placa de conversión de analógico - digital de 16 bits y 40 canales, el cual fue instalado en la computadora y fue controlado directamente por un programa desarrollado en LabView 8.0. La lectura de la placa de conversión fue mapeada en una faja de $-10 \mathrm{~V}$ a 10 $\mathrm{V}$, resultando una resolución de aproximadamente 0,305 $\mathrm{mV}$. La frecuencia de adquisición de los datos fue de 1024 $\mathrm{Hz}$, el tiempo de medición fue de $16 \mathrm{~s}$, por lo que cada medición presentó un total de 16384 datos.

\section{Resultados y Discusión}

Los coeficientes de arrastre fueron calculados para los tres tipos de ensayos realizados: 1) módulo de la torre sin antenas $\left.\left(C_{a t}\right), 2\right)$ antena VHF independiente $\left(C_{a a}\right.$ ), 3) módulo de la torre con antenas $\operatorname{VHF}\left(C_{a t a}\right)$. La fuerza de arrastre se obtuvo a partir de la expresión general representada por la ecuación 1.

$$
\mathrm{C}_{\mathrm{a}}=\frac{\mathrm{F}_{\mathrm{a}}}{1 / 2 \rho V^{2} \mathrm{~A}_{\mathrm{r}}}
$$

Donde $\left(F_{a}\right)$ es la fuerza media de arrastre, $(\rho)$ es la densidad del aire obtenida durante los ensayos, $(V)$ es la velocidad media del viento, $\left(A_{r}\right)$ es el área referencia. El área de referencia utilizada para el cálculo de los coeficientes de arrastre $\left(C_{a t}\right)$ y $\left(C_{a t a}\right)$ fue el área proyectada de una cara del módulo de la torre en la dirección normal al flujo el viento $\left(A_{t}\right)$, el valor calculado fue de $0,031 \mathrm{~m}^{2}$. El área de referencia utilizada para el cálculo del coeficiente de arrastre de la antena $\operatorname{VHF}\left(C_{a a}\right)$ fue el área frontal de la antena $\left(A_{a}\right)$, el valor calculado fue de $0,011 \mathrm{~m}^{2}$.

La tabla 1 muestra los valores de los coeficientes de arrastre obtenidos para cada tipo de ensayo. Los resultados evidencian mayores valores de coeficientes de arrastre en todos los tipos de ensayos para el flujo con alta intensidad de turbulencia (IT2) con respecto a los obtenidos para el flujo con baja intensidad de turbulencia (IT1), estos resultados coinciden con los obtenidos por Smith [15] y difieren de los resultados obtenidos por Carril Jr, Isyumov [2]. Las causas que generan estas diferencias deberán ser estudiadas en futuros trabajos. 
Los resultados de la Tabla 3 confirman que existen diferencias entre los valores de los coeficientes de arrastre del módulo de la torre sin antenas $\left(C_{a t}\right)$ y del módulo de la torre con la presencia de las antenas VHF ( $C_{\text {ata }}$ ). cálculo de la carga de viento de una torre con presencia de las antenas VHF por las vías tradicionales (sumar las fuerzas de viento de la torre y las antenas de forma aislada).

Tabla 3. Coeficientes de arrastre obtenidos.

\begin{tabular}{cccccc}
\hline & \multicolumn{4}{c}{$\mathrm{Ca}$} \\
\cline { 2 - 6 } Tipo de ensayo & \multicolumn{3}{c}{$0^{\circ}$} & \multicolumn{3}{c}{$45^{\circ}$} \\
& IT 1 & IT 2 & IT 1 & IT 2 \\
1) Módulo de torre independiente $\left(C_{a t}\right)$ & 2,13 & 2,36 & 2,53 & 2,67 \\
2) Antena VHF independiente $\left(C_{a a}\right)$ & 1,76 & 1,87 & 1,37 & 1,47 \\
3) Módulo de torre con antenas $\operatorname{VHF}\left(C_{\text {ata }}\right)$ & 2,74 & 2,98 & 3,18 & 3,37 \\
\hline
\end{tabular}

La principal inquietud está en identificar si la fuerza del viento total sobre la torre $\left(F_{T}\right)$ (ver ecuación 2) calculada a partir de la suma de la fuerza del viento sobre el módulo de la torre $\left(F_{t}\right)$ más la fuerza del viento generada por las antenas independientes $\left(F_{a}\right)$ se modifica al considerar el efecto de las antenas colocadas sobre la torre.

$$
F_{T}=F_{t}+F_{a}
$$

Para tener en cuenta el efecto de las antenas se debe multiplicar el coeficiente de arrastre de la antena independiente por un factor de interferencia $\left(F_{a}\right)$, ver ecuación 3. El factor de interferencia se define como la razón entre el coeficiente de arrastre de la antena cuando se encuentra sobre la torre $\left(\Delta C_{A q}\right)$ y el coeficiente de arrastre de la antena obtenido de forma independiente $\left(C_{a a}\right)$, ver ecuación 4 . Si el factor de interferencia es diferente de 1 indica la necesidad de considerar el efecto de interferencia de las antenas colocadas sobre la torre, de lo contrario se estaría subestimando o sobrestimando el valor de la fuerza total de la carga de viento.

$$
\begin{aligned}
& F_{T}=q \cdot \mathrm{C}_{\mathrm{at}} \cdot \mathrm{A}_{\mathrm{t}}+q \cdot \mathrm{C}_{\mathrm{aa}} \cdot \mathrm{f}_{\mathrm{a}} \cdot \mathrm{A}_{a} \\
& f_{a}=\frac{\Delta C_{A a}}{C_{a a}}=\left(\frac{F_{\text {torre }+ \text { antenas }}-F_{\text {torre }}}{1 / 2 \rho V^{2} A_{a}}\right) / C_{a a}
\end{aligned}
$$

Los factores de interferencia obtenidos cuando la torre presenta cuatro antenas VHF colocadas en cada una de las caras se muestran en la Tabla 4. Estos resultados solo son válidos para la disposición de antenas ensayadas en este trabajo y denotan que no se debe generalizar el
Tabla 4. Factores de interferencia para las antenas VHF

\begin{tabular}{ccc}
\hline \multirow{2}{*}{ Dirección } & \multicolumn{2}{c}{$f_{a}$} \\
& IT1 & IT2 \\
\hline 0 & 0,99 & 0,96 \\
45 & 1,38 & 1,34 \\
\hline
\end{tabular}

\section{Conclusiones}

De los resultados se evidencia que la presencia de las antenas VHF sobre la torre incrementa en más de un $60 \%$ el valor del coeficiente de arrastre y por lo tanto de la fuerza de viento total sobre la estructura.

Los mayores incrementos del coeficiente de arrastre se obtienen para la dirección de viento de 45으, o sea, cuando se evidencia un aumento del área expuesta al viento entre la relación torre-antena VHF.

Para la dirección de viento 0 잉 los valores del factor de interferencia son aproximadamente 1, esta información indica que para esa dirección la fuerza del viento total se puede calcular por las vías tradicionales. Para la dirección de $45^{\circ}$ los factores de interferencia superan en más de un $30 \%$ la unidad; por lo que debe tenerse en cuenta el efecto de interferencia que representan las antenas VHF cuando están colocadas sobre la torre, de lo contrario se estaría subestimando el valor de la fuerza total de la carga de viento.

De forma general, se demuestra que no siempre es válido aplicar el principio de superposición de efectos derivado del enfoque de cálculo tradicional; por lo que se 
recomienda emplear la vía experimental siempre que sea posible.

\section{Agradecimientos}

Los autores agradecen el financiamiento proporcionado por CAPES, Fundación de Coordinación para el Perfeccionamiento del Personal de Nivel Superior, Brasil y al personal que labora en el Túnel de Viento "Prof. Joaquim Blessmann", que hicieron posible la realización de este trabajo.

\section{Referencias Bibliográficas}

[1] Cengel, Y. and J.M. Cimbala.: "Fluid Mechanics, Fundamentals and Applications", McGraw-Hill, New York, 2006.

[2] Carril Jr, C.I.F., Isyumov, N. and Brasil, R.M.L.R.F.: "Experimental study of the wind forces on rectangular latticed communication towers with antennas". Journal of Wind Engineering and Industrial Aerodynamics, Vol. 91, N. 8 (2003) 1007 1022.

[3] Holmes, J.D., Banks, R.W. and Roberts, G.: "Drag and aerodynamic interference on microwave dish antennas and their supporting towers". Journal of Wind Engineering and Industrial Aerodynamics, Vol. 50 (1993) 263-269.

[4] ESDU, Item 81028: Lattice Structures. Part 2: Mean Fluid Forces on Tower-like Space Frames, E.S.D. Unit, Editor, London (1993).

[5] Martín, P., et al.: "Experimental study of the effects of dish antennas on the wind loading of telecommunication towers". Journal of Wind Engineering and Industrial Aerodynamics, Vol. 149, N. 2 (2016) 40-47.

[6] Vickery, B.J.: The aeroelastic modelling of chimneys and towers, in Workshop on Wind Tunnel Modeling for Civil Engineering Applications, Gaithersburg, Maryland, Cambridge University Press, USA (1982).

[7] Holdo, A.E.: "Reynolds number effects on lattice structures forming part of a wind tunnel model". Journal of Wind Engineering and Industrial Aerodynamics, Vol. 45, N. 2 (1993) 229-238.

[8] Mitchell, J. W., Pritchard, P. J., McDonald, A. T., Fox, R. W., and Mitchell, J. W.: "Fox and McDonald's introduction to fluid mechanics", 8th Edition, John Wiley \& Sons, Inc, USA, 2011.

[9] Holmes, J.D.: "Wind loading of structures", ed. T.F. e-Library, ed. T. Francis, New York, 2007.
[10] EC 3: Part 3-1, Eurocode 3: Design of steel structures - Part 3-1: Towers, masts and chimeneys-Towers and masts, Belgium (2007).

[11] TIA/EIA-222-G: Structural standards for Steel Antenna Towers and Antenna Supporting Structures, Telecommunications Industry Association, USA (2005).

[12] RYMSA. Catálogos de Antenas RYMSA, edición 8. www.rymsa.com 2013 Febrero 2013].

[13] NC-285: Carga de viento. Método de cálculo, Oficina Nacional de Normalización (NC), Cuba (2003)

[14] Blessmann, J.: "The boundary layer tv-2 wind tunnel of the UFRGS". Journal of Wind Engineering and Industrial Aerodynamics, Vol. 10, N. 1 (1982) 231248.

[15] Smith, B.W.: "Communication structures", First ed, ed. A. Thomas Telford, London, Great Britain, 2007. 


\section{REVISTA TECNICA}

DE LA FACULTAD DE INGENIERIA

UNIVERSIDAD DEL ZULIA

Vol. 42. N³, Septiembre - Diciembre 2019, pp. 104 -151

Esta revista fue editada en formato digital y publicada en Septiembre de 2019, por el Fondo Editorial Serbiluz, Universidad del Zulia. Maracaibo-Venezuela

www.luz.edu.ve

www.serbi.luz.edu.ve

produccioncientifica.luz.edu.ve 